\title{
Automatic Exact Histogram Specification for Contrast Enhancement and Visual System Based Quantitative Evaluation
}

\author{
Debashis Sen, Member, IEEE, and Sankar K. Pal, Fellow, IEEE
}

\begin{abstract}
Histogram equalization, which aims at information maximization, is widely used in different ways to perform contrast enhancement in images. In this paper, an automatic exact histogram specification technique is proposed and used for global and local contrast enhancement of images. The desired histogram is obtained by first subjecting the image histogram to a modification process and then by maximizing a measure that represents increase in information and decrease in ambiguity. A new method of measuring image contrast based upon local band-limited approach and center-surround retinal receptive field model is also devised in this paper. This method works at multiple scales (frequency bands) and combines the contrast measures obtained at different scales using $L^{p}$-norm. In comparison to a few existing methods, the effectiveness of the proposed automatic exact histogram specification technique in enhancing contrasts of images is demonstrated through qualitative analysis and the proposed image contrast measure based quantitative analysis.
\end{abstract}

Index Terms-Ambiguity measures, beam theory, center-surround retinal receptive field, contrast enhancement, exact histogram specification, fuzzy sets, local band-limited contrast.

\section{INTRODUCTION}

C ONTRAST enhancement is an important image processing technique that makes various contents of images easily distinguishable through suitable increase in contrast. Histogram specification is a method where contrast enhancement is obtained by suitably changing the image histogram into a desired one. Exact histogram specification [1] guarantees that the histogram of the image obtained after enhancement is almost exactly the desired one. However, there does not exist any obvious choice for the desired histogram [1]. Mostly, the desired histogram has been considered as uniform (histogram equalization). A few times the desired image histogram has been considered as the one which makes the histogram of perceived brightness uniform (histogram hyperbolization [2]). In certain cases, the desired histogram has been considered as unimodal/bimodal Gaussian or exponential depending upon the underlying application.

Manuscript received March 16, 2010; revised July 28, 2010; accepted September 15, 2010. Date of publication October 04, 2010; date of current version April 15, 2011. The work of S. K. Pal was supported by the Government of India under the J. C. Bose National Fellowship. The associate editor coordinating the review of this manuscript and approving it for publication was Prof. Peyman Milanfar.

The authors are with the Center for Soft Computing Research, Indian Statistical Institute, Kolkata, 700108 India (e-mail: dsen_t@isical.ac.in; sankar@isical.ac.in).

Digital Object Identifier 10.1109/TIP.2010.2083676
The exact histogram specification scheme of [1] is based upon strict ordering among image pixels via calculation of local mean values for contrast enhancement. Exact histogram equalization, as in [1], guarantees that the histogram of the image obtained after enhancement is uniform (ignoring the possibility of an insignificant error). Therefore, the process of exact histogram equalization is a process of information entropy maximization, and it increases the contrast of an image by maximizing information retrievable from the image.

In this paper, we propose an automatic exact histogram specification technique. The desired histogram is obtained by first subjecting the image histogram to a modification process that increases the overall discriminablity among samples in the histogram, and then maximizing a measure that represents increase in information entropy [3] and decrease in average image ambiguity [4]. The aforesaid approach is based upon the finding that increase in information entropy and decrease in average image ambiguity, which are indicators of contrast enhancement, are contradictory. The proposed exact histogram specification technique is used for global and local contrast enhancement of grayscale images.

Global and local contrast enhancement performed using the proposed automatic exact histogram specification technique is compared to the usage of exact histogram equalization [1] and to a few related state of the art existing techniques. Qualitative and quantitative evaluation of contrast enhancement performance is considered, and the effectiveness of the proposed exact histogram specification technique is demonstrated.

In order to perform quantitative analysis, a new method of measuring image contrast based upon local band-limited approach [5] and center-surround retinal receptive field model [6] is devised in this paper. The concept of quantifying local contrast in complex images given in [5] and antagonistic center-surround models such as the difference of Gaussian (DoG) [7] are brought together in our approach of image contrast measurement. Evidences from physiological studies corresponding to contrast sensitivity in achromatic and color vision [8]-[10], and sub- and supra-threshold contrast perception [11], [12] are considered in the proposed approach. Considering the existence of multiple frequency channels in the visual system [13], the proposed method works at multiple scales (frequency bands) and combines the contrast measures obtained at different scales using $\mathrm{L}^{\mathrm{p}}$-norm. Suitable functions involving image contrast measured using the proposed approach are employed for evaluation of contrast enhancement. 


\section{Proposed Automatic Exact Histogram SPECIFICATION METHOD}

As mentioned in Section I, the process of exact histogram equalization is a process of information entropy maximization. In this section, we propose an automatic exact histogram specification technique that:

— increases the overall discriminablity among samples in the histogram that correspond to pixels in the underlying image;

- maximizes a measure that represents increase in information entropy and decrease in average image ambiguity.

Authors in [14] have proposed segmentation via a density modification technique, which increases the overall discriminablity among samples in a feature space that correspond to pixels in the underlying image, using a fuzzy set theory based system devised with cue from beam theory of solid mechanics. In the process of determining the desired histogram (histogram to be specified) from the original histogram (histogram of the underlying image before enhancement), we consider the concept of increasing the overall discriminablity among samples given in [14].

In [4], it is pointed out that average ambiguity in an image reduces with increase in the distinguishablity of various parts of the image and it is shown that the average image ambiguity measure in [4] can be considered as an indicator of contrast enhancement. Hence, in addition to increase in information entropy, which is considered in the classical technique of histogram equalization, we also consider decrease in average image ambiguity in the proposed histogram specification technique. Increase in information entropy and decrease in average image ambiguity are found to be contradictory and, hence, we devise a optimal tradeoff approach to achieve both satisfactorily.

\section{A. Determination of the Desired Histogram From the Original Histogram}

1) Increasing the Overall Discriminability Among Samples in the Histogram: In the proposed approach of determining the desired histogram from the original histogram, the first step is a histogram modification process. We increase the overall discriminablity among samples in the histogram that correspond to pixels in the underlying image through the modification process. The histogram modification process, which is based upon an altered version of the density modification approach given in [14], is described here. Without loss of generality, we shall describe the process assuming a gray-level histogram of a grayscale image.

Let $O$ be the gray-level histogram of the grayscale image under consideration. We first normalize $O$ as follows:

$$
\bar{O}(i)=\frac{O(i)}{\sum_{z \in G} O(z)}, \quad i \in G
$$

where $G$ is the universal set of gray levels. The quantity $\bar{O}(i)$ gives the probability of occurrence of gray value $i$ in the image under consideration.

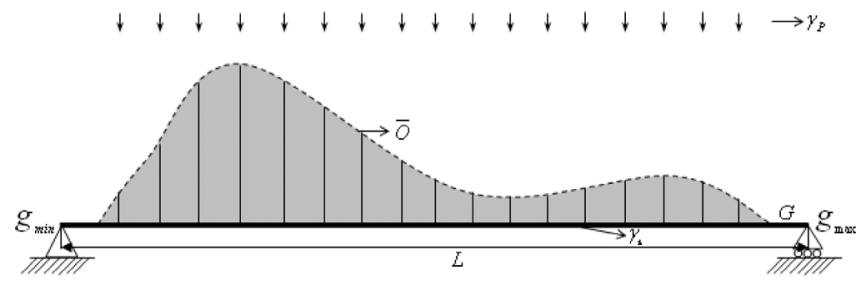

Fig. 1. Setup for the proposed histogram modification.

We shall now consider some concepts from beam theory of solid mechanics. Consider a solid whose shape is given by $\bar{O}$. Let the solid be placed over a beam of uniform (along the length) height and width. Let us assume that the entire uniform beam and the entire solid are made out of the same material. Consider that the beam is rested upon two pivots at the two ends. Let the two ends refer to the minimum $\left(g_{\min }=\min _{z \in G} z\right)$ and maximum $\left(g_{\max }=\max _{z \in G} z\right)$ gray values. Let us consider that a uniform (along the length) force $\left(\gamma_{P}\right)$, say due to gravity, is always acting upon the uniform beam. Fig. 1 gives the pictorial representation of the aforesaid setup. The total force acting on the beam is $P(i)=\bar{O}(i)+\gamma_{P}$ and we consider $\gamma_{P}$ as $\left(\sum_{z \in G} O(z)\right)^{-1}$, which represents one unit (force) with respect to $\bar{O}$.

Once $\bar{O}(i)$ is obtained from $O(i)$ using (1), we calculate the bending moment $B(i)$ due to the force $P(i)$ at a gray value $i \in G$ (Fig. 1) using Euler-Bernoulli beam theory as follows:

$$
B(i)=(R \times l)-(C P(i) \times(l-C G(i))), \quad l=i-g_{\min } .
$$

In the previous equation, $C P\left(i_{1}\right)$ stands for the total (cumulative) force between the gray values $g_{\min }$ and $i$, and it is given as

$$
C P(i)=\sum_{z=g_{\min }}^{i} P(z)=\sum_{z=g_{\min }}^{i}\left(\bar{O}(z)+\gamma_{P}\right) .
$$

The symbol $C G(i)$ stands for the center of gravity between the gray values $g_{\min }$ and $i$, and it is given as

$$
C G(i)=\frac{1}{C P(i)} \sum_{z=g_{\min }}^{i}\left(z-g_{\min }\right) P(z) .
$$

In (2), $R$ is the reactive force at the pivot at $g_{\min }$ and it is calculated as

$$
\begin{aligned}
& R=C P\left(g_{\max }\right) \times\left[\frac{L-C G\left(g_{\max }\right)}{L}\right] \\
& \text { where } L=g_{\max }-g_{\min } \text {. }
\end{aligned}
$$

Now, consider the following function obtained by normalizing the bending moment $B$ :

$$
\mu(i)=\frac{B(i)}{\max _{z \in G} B(z)}, \quad i \in G .
$$

It is shown in [14] that $\mu$ can be considered as a membership function corresponding to a fuzzy set defined in $G$ and the value of the membership function $\mu$ at a gray value $i$ represents a property "farness of the gray value from the nearest among $g_{\min }$ and 
$g_{\text {max }}$." Note that, the aforesaid terms "farness" and "nearest" are inherently defined in $\mu$. The gray value where $\mu$ takes a value of unity, is the gray value that is equally far from both $g_{\min }$ and $g_{\text {max }}$. A gray value smaller (larger) than that gray value is nearer to $g_{\min }\left(g_{\max }\right)$ compared to $g_{\max }\left(g_{\min }\right)$.

As the groups of samples in the histogram associated with $g_{\min }$ and $g_{\max }$ are the most discriminable ones from each other, the aforesaid property $\mu$ at a gray value gives the farness of the samples in the histogram associated with that gray value from the nearest among the mutually most discriminable groups of samples in the histogram. Therefore, the property $\mu$ can be used to modify the histogram $O$ such that the overall discriminability among samples in the histogram is increased.

It is evident from (2) and (6) that $\mu$ is dependent upon the histogram $O$. However, one might like to have the property 'farness of a gray value from the nearest among $g_{\min }$ and $g_{\max }$ ' such that it depends only upon the gray value $(i \in G)$ and not on the histogram $(O)$. Note that, if the value of $\gamma_{P}$ is considered such that $\gamma_{P} \gg \max _{z \in G} \bar{O}(z)$, the membership function approximately becomes independent of $O$ and depends only upon the underlying gray value.

Now, we know that the bending moment $B$ refers to a membership function $\mu$, which represents a useful property "farness of a gray value from the nearest among $g_{\min }$." We shall now modify the histogram $O$ using concepts from beam theory, where the bending moment $B$ will be appropriately considered.

The curvature due to the bending moment $B(i)$ at a gray value $i \in G$ is as follows:

$$
\rho(i)=\frac{B(i)}{\iota(i)}
$$

where $\iota(i)$ is the moment of inertia (opposing the bending) at $i$, which is calculated as

$$
\iota(i)=\sum_{k=0}^{\bar{O}(i)+\gamma_{\iota}}(k-c(i))^{2}
$$

where $c(i)$ denotes the centroid of the solid and the beam taken together at the gray value $i$ and it is given by $0.5 \times\left(\bar{O}(i)+\gamma_{\iota}\right)$. We consider that the height of the beam $\gamma_{\iota}$ (see Fig. 1) equals $\left(\sum_{z \in G} O(z)\right)^{-1}$, which represents one unit with respect to $\bar{O}$.

However, it is observed that some $\bar{O}$ may correspond to solids which when considered would result in curvatures $(\rho(i))$ that are very large at a few gray values, making the curvatures at other gray values insignificant. We find that such situations are unfavorable for increasing the overall discriminability among samples in the histogram, as many gray values would be ignored during the modification process. Therefore, we consider the following measure instead of $\rho$ at a gray value $i \in G$ :

$$
\rho(i)=\frac{B(i)}{\iota(i)+\max _{z \in G} \iota(z)}
$$

and then normalize $\rho$ as follows:

$$
\bar{\rho}(i)=\frac{\dot{\rho}(i)}{\sum_{z \in G} \hat{\rho}(z)}, \quad i \in G .
$$

Once the value of $\bar{\rho}(i)$ for all $i \in G$ has been obtained, we perform the following operation:

$$
\theta(i)=\max \left(\left[\max _{z \in G} \bar{\rho}(z)-\bar{\rho}(i)+D \times \max _{z \in G} \bar{\rho}(z), 0\right]\right)
$$

where $D$ is a real value in the interval $(-1, \infty)$. We then normalize $\theta$ as follows:

$$
\bar{\theta}(i)=\frac{\theta(i)}{\sum_{z \in G} \theta(z)}, \quad i \in G .
$$

We consider the quantity $\bar{\theta}(i)$ as the probability of occurrence of gray value $i$ in the image having the modified histogram. The modified histogram, say $O^{M}$ is then determined from $\bar{\theta}$ such that the number of samples in the modified histogram equals that in the original histogram, as follows:

$$
O^{M}(i)=\left\lfloor\bar{\theta}(i) \times \sum_{z \in G} O(z)\right\rfloor, \quad i \in G .
$$

The discrepancy $\epsilon=\sum_{z \in G} O(z)-\sum_{z \in G} O^{M}(z)$ is then omitted by adding one sample to each of the $\epsilon$ largest bins (number of occurrences) in $O^{M}$.

It is ascertained in [14] that considering the aforesaid quantity $O^{M}$ as the modified histogram is appropriate as the overall discriminablity among samples is increased by the explained histogram modification process.

2) Determination of the Parameter D-Tradeoff Between Increase in Information Entropy and Decrease in Average Image Ambiguity: Consider the expression of $\theta(i)$ from (11). As mentioned earlier, $D$ is a real value in the interval $(-1, \infty)$. From Section II-A.1, it can be easily deduced that $\bar{\rho}(i)=0$ only when $i=g_{\min }$ or $i=g_{\max }$. Therefore, it is evident from (11) that $\theta\left(g_{\min }\right)=\theta\left(g_{\max }\right)$ and when $D$ is close enough to $-1, \theta(i)$ is nonzero only at $i=g_{\min }$ and $i=g_{\max }$. In such a case, all the samples in the modified histogram $O^{M}$ would be at $g_{\min }$ and $g_{\max }$. Now as $D \rightarrow \infty$, it is evident from (11) that the values of $\theta(i) \forall i$ tends to be almost the same. In such a case, all the samples in the modified histogram $O^{M}$ tend to be more or less equally distributed (uniform) at all gray values. From the previous discussion, it is evident that the parameter $D$ is a very significant one. We devise an approach here to determine the parameter $D$ in an optimal sense. The modified histogram $O^{M}$ that uses the optimal value of $D$ is the desired histogram in our novel exact histogram specification technique.

As mentioned earlier, when $D \rightarrow \infty, O^{M}$ tends to be uniform. It is well understood and also empirically observed that information entropy [3] calculated from $O^{M}$ increases as $O^{M}$ tends to be uniform [8]. Therefore, information entropy due to $O^{M}$ increases as $D \rightarrow \infty$. From the theory of histogram equalization [8] we know that information entropy maximization is a potent way of contrast enhancement. However, it is also well known that information entropy maximization may cause artifacts such as washed-out effect and clutter intensification in the underlying image [15].

On the other hand, when $D \rightarrow-1$, the number of samples in $O^{M}$ tends to be nonzero only at $g_{\min }$ and $g_{\max }$. Therefore, as also evident from the earlier discussion, information entropy due to $O^{M}$ decreases as the value of $D$ decreases. However, we 
find that the average image ambiguity measure [4] based upon grayness ambiguity [4] calculated from $O^{M}$ also decreases as $D \rightarrow-1$. It is known that minimization of average image ambiguity or other measures of image ambiguity is a potent way of contrast enhancement [4]. The effect of the aforesaid minimization process in the underlying histogram is such that the samples in it get concentrated near two highly distinct gray values. Therefore, although the overall contrast of the underlying image would be intensified, the process may lead to loss of details.

From the previous explanation, we see that increase in information entropy and decrease in average image ambiguity calculated from $O^{M}$ are contradictory in the context of change in the parameter $D$. However, both increase in information entropy and decrease in average image ambiguity are desirable traits in contrast enhancement of images. Therefore, the requirement is to perform a tradeoff between increase in information entropy and decrease in average image ambiguity in order to determine the parameter $D$. Such a tradeoff would constrain both increase of information entropy and decrease of average image ambiguity, which would diminish the aforesaid disadvantages of both. We consider the maximization of the following measure to determine $D$ :

$$
\digamma=(1-A I A) \times \bar{H}
$$

In the previous equation, $\bar{H}$ represents the Shannon's information entropy $H$ [3] normalized such that $\bar{H} \in[0,1]$ and $H$ is calculated from $O^{M}$. Average image ambiguity is represented by $A I A$, where $A I A \in[0,1]$. The computation of $A I A$ is based upon grayness ambiguity calculated from $O^{M}$, which has been elaborately explained in [4].

As mentioned earlier, we intend to obtain the optimal value of $D$ as

$$
D_{\mathrm{opt}}=\arg \max _{D \in(-1, \infty)} \digamma \cdot
$$

We consider that $O^{M}$ [see (13)] as the desired histogram in our exact histogram specification technique, which is obtained considering $D=D_{\mathrm{opt}}$ in (11). We have carried out the process of determination of desired histogram considering several original histograms and we have made the following observations:

- both $A I A$ and $\bar{H}$ increase with increase in $D$;

- rate of increase of both $A I A$ and $\bar{H}$ decrease with increase in $D$;

- with respect to increase in $D$, initially, the rate of increase of $\bar{H}$ is more than that of $A I A$; Later, the rate of increase of $\bar{H}$ becomes less than that of $A I A$;

- $A I A$ and $\bar{H}$ as functions of the parameter $D$ fit the expressions $C_{1}\left(-\exp (-\kappa D)+C_{2}\right)$ and $C_{3}\left(-\exp (-\tau D)+C_{4}\right)$, respectively, where $\tau>\kappa$ and $C_{1}, C_{2}, C_{3}$ and $C_{4}$ are arbitrary constants.

From the expression of $\digamma$ in (14) and the first three aforesaid observations, it can be easily inferred that $\digamma$ as a function of $D \in(-1, \infty)$ would have a unique maximum. The same inference can be mathematically deduced considering the fourth observation. The value of $D$ at the solitary maximum is $D_{\text {opt }}$ and, hence, searching for $D_{\text {opt }}$ in the entire interval $(-1, \infty)$ is not required.

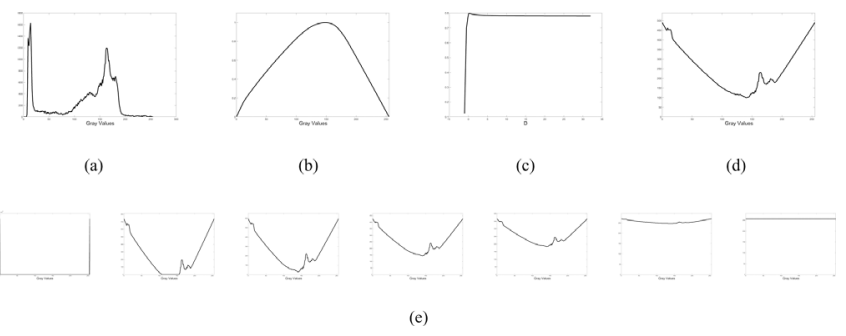

Fig. 2. Graphical representation of the determination of the desired histogram from the original histogram. (a) Original Histogram. (b) Membership function $\mu$. (c) $\digamma$ (D). (d) $O^{M}$ when $D=D_{\mathrm{opt}}$. (e) $O^{M} \mathrm{~s}$ with $D=-.99,-.1, .05, .1,1,10,1000$ (left to right).

The nature of dependency of $O^{M}$ on the value of $D$ and the behavior of $\digamma$ as a function of $D$, which are explained in Section II-A.2, are shown graphically in Fig. 2. Note the unique maximum of $\digamma$ as a function of $D$ in Fig. 2(c), and the near extreme cases of $O^{M}$ when $D$ is very near to -1 and when $D$ takes a very large value in Fig. 2(e). Observe in Fig. 2 that as explained in Section II-A.1, the overall discriminability among samples in $O^{M}$ has increased compared to the original histogram as more samples in $O^{M}$ are nearer to any of the two mutually most discriminable groups of samples, which are at the minimum and maximum gray values. Notice [see Fig. 2(a) and (d)] the signature of the original histogram $O^{M}$ in the form of valleys and peaks. The property represented by the membership function $\mu$ stated in Section II-A.1 is evident from Fig. 2(b).

\section{B. Implementation of Exact Histogram Specification}

Once the desired histogram is determined using the proposed approach, we implement exact histogram specification as described in [1]. Note that the proposed approach of desired histogram determination is a fully automatic one and, hence, we have presented a novel automatic exact histogram specification technique, which can be used on global, local and partial (sub) histograms to perform global, mean brightness preserving, dynamic and local contrast enhancement of images.

\section{Retinal Visual System Based IMAge Contrast MEASUREMENT AND QUANTITATIVE EVALUATION OF CONTRAST ENHANCEMENT}

Quantitative evaluation of contrast enhancement is not trivial, as there do not exist any universally accepted measure of contrast or ideally enhanced images as references. Measures of dispersion (local and global) such as variance, standard deviation and entropy have been used to evaluate contrast enhancement. Contrasts calculated according to Weber's and Michelson's definition [5], [8] have also been employed for the same. Contrast enhancement has been evaluated using measures based upon local gradient magnitude such as the Tenengrad value [15], and also using average distance between pixels on the gray scale [15]. These aforesaid measures hardly represent the actual image contrast viewed by the eye.

Quantitative evaluation of contrast enhancement should be based upon appropriate measurement of contrast at all image pixels. Any reasonable measure of contrast should be at least crudely tuned according to the retinal visual system and such a 
measure would then probably be more credible and universally acceptable. In this section, we present a novel approach of quantitative evaluation of contrast enhancement using a new method of image contrast measurement based upon several available studies on the retinal visual system.

\section{A. Image Contrast Measurement}

1) Contrast Due to Achromatic Signal: We base our method of contrast measurement on Peli's [5] local band-limited approach. As considered in [5], we assume that contrast at a grayscale image pixel should be expressed as the dimensionless ratio of the local change and the local average. Analysis on the applicability of Weber's and Michelson's contrast definition to complex images has been carried out in [5] and the related drawbacks were addressed leading to a definition of local band-limited contrast for complex images. The local band-limited contrast is defined in [5] as

$$
c(x, y)=\frac{\beta(x, y)}{\lambda(x, y)}
$$

where

$$
\begin{aligned}
& \beta(x, y)=f(x, y) * b(x, y) \\
& \lambda(x, y)=f(x, y) * l(x, y) .
\end{aligned}
$$

In (18), $f$ is the grayscale image under consideration, $b$ is a bandpass filter, $l$ is a low-pass filter such that it passes all energy below the passband of $b$ and $*$ represents the convolution operator.

Let us now briefly consider the phenomenon of perception in the retina of an eye. Photoreceptor cells, which are capable of phototransduction, are present in the retina and two main types of photoreceptors are referred to as cones and rods [6]. The signals generated by phototransduction in the retina pass through such pathways that both excitatory and inhibitory fields are generated [6]. It is widely accepted that the excitatory and the inhibitory fields organize in the retina such that center-surround retinal (ganglion) receptive fields are formed, where the center and the surround fields are antagonistic [see Fig. 3(a)]. The center-surround organization is called on-center when the center is excitatory $(+)$ and the surround is inhibitory $(-)$ and it is called offcenter in the other case. Now, it is shown in [7] that the difference of Gaussian (DoG) can be used to model center-surround retinal receptive field appropriately in the case of achromatic signal. The application of DoG based center-surround model on a grayscale image, which represents an achromatic signal, is represented as

$$
O(x, y)=C(x, y)-S(x, y)
$$

where

$$
\begin{aligned}
C(x, y) & =f(x, y) * g_{1}(x, y) \\
S(x, y) & =f(x, y) * g_{2}(x, y) .
\end{aligned}
$$

In (19)-(21), $O$ is the output of the DoG based center-surround operator, $C$ and $S$ represent the signals from the center and the surround, respectively, and $g_{1}$ and $g_{2}$ represent the two Gaussian functions of the form $\exp \left((-1) /\left(2 \sigma^{2}\right)\left(x^{2}+y^{2}\right)\right)$ that make up

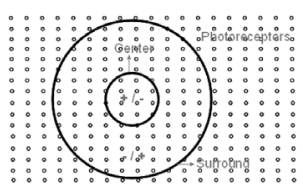

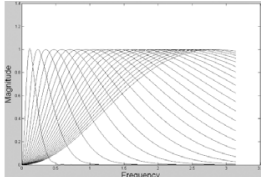

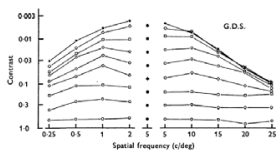

Fig. 3. Pictorial representation of some vision related phenomena of the retina during perception of an achromatic signal. (a) Center-surround retinal receptive field. (b) Multiple bandpass channels. (c) Sub- and supra-threshold contrast (taken from [11])].

the DoG operator $g_{1}-g_{2}$. Note that the standard deviation $(\sigma)$ of $g_{1}$ is smaller than that of $g_{2}$.

We shall now bring together the concept of band-limited local contrast in complex images [5] and the concept of DoG based center-surround retinal receptive field model [7]. Observe that the DoG operator $g_{1}-g_{2}$ is a bandpass filter and both the Gaussian functions $g_{1}$ and $g_{2}$ are low-pass filters. Another interesting observation is that $g_{2}$ is a low-pass filter that passes all energy below the passband of the DoG operator $g_{1}-g_{2}$. Therefore, we can readily use the output $(O)$ of the bandpass filter (DoG operator) $g_{1}-g_{2}$ and the output $(S)$ of the low-pass filter $g_{2}$ in the local band-limited contrast definition given in (16) as follows:

$$
c(x, y)=\frac{\beta(x, y)}{\lambda(x, y)}=\frac{O(x, y)}{S(x, y)} .
$$

The quantity $|c(x, y)|$ gives a local band-limited contrast measure at the image pixel $(x, y)$. In the calculation of $c(x, y)$, we consider that the standard deviations of $g_{1}$ and $g_{2}$ are related as $\sigma_{g_{2}}=M \times \sigma_{g_{1}}$, where $M$ is an arbitrary real value greater than unity.

Now, evidence of presence of multiple spatial frequency selective channels in the retina has been found [13]. The measure $|c(x, y)|$ is obtained from a single spatial frequency channel/ band. We consider the value of $M=3$ and in order to mimic the presence of multiple frequency channels in the retina, we consider multiple values of $\sigma_{g_{1}}$. Multiple standard deviation values of $g_{1}$ give multiple spatial bandpass filters $g_{1}-g_{2}$ having different passbands and, hence, we get multiple $|c(x, y)|$ values which we represent as $\left|c_{\sigma_{g_{1}}}(x, y)\right|$. We consider 24 values of $\sigma_{g_{1}}$ as follows:

$$
\sigma_{g_{1}}=\frac{2 \log \left(\frac{1}{M^{2}}\right)}{\nu^{2}\left(1-M^{2}\right)}, \quad \nu=\frac{72 \pi}{80}, \frac{69 \pi}{80}, \cdots, \frac{6 \pi}{80}, \frac{3 \pi}{80}
$$

where $\nu \in[0, \pi]$ is the frequency at which the passband of the underlying bandpass filter peaks (center/peak frequency). Note that, $\pi(2 \pi \times(1 / 2))$ corresponds to the maximum possible spatial frequency $(1 / 2)$ cycles per pixel width and the unit of $\sigma_{g_{1}}$ is pixel width. The passbands (frequency channels) of 1-D equivalents of the 24 bandpass filters (normalized such that the value at peak frequency is unity) corresponding to the 24 values of $\sigma_{g_{1}}$ are shown in Fig. 3(b). Note that the application of the DoG operator $g_{1}-g_{2}$ on a grayscale image considering multiple standard deviation values of $g_{1}$ in order to measure contrast essentially means that the contrast is measured at multiple scales. In order to get a single contrast value at a pixel $(x, y)$, we need to 
combine the contrast measures $\left(\left|c_{\sigma_{g_{1}}}(x, y)\right|\right)$ obtained at multiple scales.

In order to perform the aforesaid combination, we consider findings from the study of sub- and supra-threshold contrast perception reported in [11]. While subthreshold contrast perception corresponds to contrast sensitivity at contrast levels near to the minimum contrast required for detection of a pattern, supra-threshold contrast perception corresponds to contrast sensitivity at much higher contrast levels. In subthreshold contrast perception, it has been found that contrast sensitivity is lower when the underlying pattern has low and high spatial frequencies compared to when the pattern has spatial frequencies in between low and high. Whereas, in supra-threshold contrast perception, contrast sensitivity characteristics show very little variation across spatial frequencies [11]. The illustration in Fig. 3(c) reproduced from [11] demonstrates the aforesaid phenomena. We find that $\mathrm{L}^{\mathrm{P}}$-norm can be used to perform the combination of all the $\left|c_{\sigma_{g_{1}}}(x, y)\right| \mathrm{s}$ in such a way that the aforesaid phenomena about sub- and supra-threshold contrast perception are mimicked. We combine the measures $\left|c_{\sigma_{g_{1}}}(x, y)\right|, \forall \sigma_{g_{1}}$ as follows:

$$
c_{L}(x, y)=\left(\sum_{\sigma_{g_{1}}}\left(P^{-1} \times\left|c_{\sigma_{g_{1}}}(x, y)\right|\right)^{p}\right)^{\left(\frac{1}{p}\right)}
$$

where $p \geq 1$ and

$$
P=\exp \left(-\frac{\log \left(\frac{1}{M^{2}}\right)}{1-M^{2}}\right)-\exp \left(-\frac{\log \left(\frac{1}{M^{2}}\right)}{1-M^{2}} \times M^{2}\right) \text {. }
$$

In (25), the normalization by $P$ ensures that the underlying passband's magnitude value at peak frequency is unity [see Fig. 3(b)]. The measure $c_{L}(x, y)$ gives the contrast measure of the grayscale image $f$, which represents an achromatic signal, at a pixel $(x, y)$. When $p=1\left(\mathrm{~L}^{1}\right.$ norm $)$ is considered in (24), the subthreshold contrast perception is best mimicked and when $p=\infty$ (maximum norm) is considered in (24), the supra-threshold contrast perception is best mimicked. Fig. 4(a) shows an image having sinusoidal pattern with varying magnitude (across rows) and spatial frequency (across columns), and the contrast measure $c_{L}$ obtained at all pixels using different values of $p$, and Fig. 4(b) shows their corresponding 1-D profiles across columns. Observe the cases of $p=1$ and $p=2$ in Fig. 4(b), where contrast sensitivity is higher when the spatial frequency is in between low and high, and the cases of $p=15$ and $p=\infty$, where contrast sensitivity hardly varies across spatial frequencies. Hence, it is evident in Fig. 4 that with increase in the value of $p$, the contrast sensitivity characteristics gradually changes from that of subthreshold contrast perception to supra-threshold contrast perception. Contrast levels in an image may vary from the minimum contrast level required for detection of a pattern to much higher contrast levels. Therefore, the choice of $p$ in (24) is not an obvious one and any analysis based upon $c_{L}(x, y)$ should at least involve the extreme cases $p=1$ and $p=\infty$.

2) Contrast Due to Chromatic Signals: As mentioned in Section III-A.1, signals generated by phototransduction in the retina create excitatory and inhibitory fields, which organize such that center-surround retinal receptive fields are formed
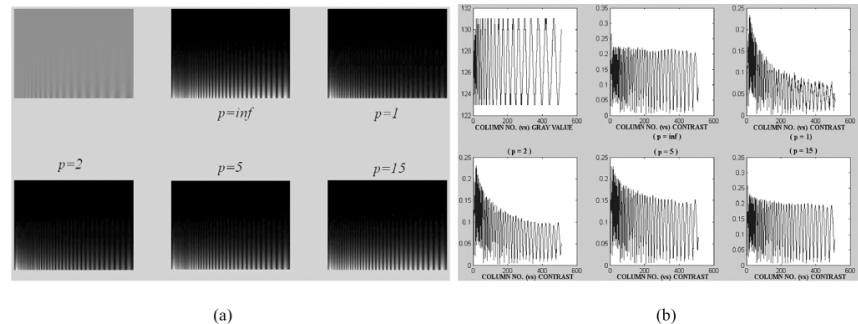

(a)

(b)

Fig. 4. Contrast measures obtained in an image, which has sinusoidal pattern with spatial frequency decreasing from left to right, considering different values of $p ; p=\infty, 1,2,5,15$ [top-middle to bottom-right]. (a) Image with sinusoidal pattern and its different contrast measures (normalized to the range $[0,1]$ ) at all pixels. (b) 1-D gray value and contrast profiles across columns.

with antagonistic center and surround. In the case of chromatic signals, the center-surround retinal receptive fields are formed in color-opponent organization [6]. The color-opponent organization of center-surround retinal receptive field is characterized by the red, green, blue and yellow components of color. A center-surround retinal receptive field in color-opponent organization would be any one of the following four; a red component center with an antagonistic green component surround, a green component center with an antagonistic red component surround, a blue component center with an antagonistic yellow component surround, a yellow component center with an antagonistic blue component surround. During perception of chromatic signals, all the aforesaid four types of center-surround retinal receptive fields are formed in the retina.

We now need an operator that mimics the center-surround retinal receptive field in color-opponent organization for application on color components of color images to calculate contrast. We consider CIE L*a* $b^{*}$ color components (CIE standard illuminant D65), where $\mathrm{L}^{*}$ represents lightness (achromatic component), $a^{*}$ represents the red-green opponent color component (red-positive, green-negative) and $b^{*}$ represents yellow-blue opponent color component (yellow-positive, blue-negative) [8]. The operator mimicking the center-surround retinal receptive field in color-opponent organization would be applied on the $\mathrm{a}^{*}$ and $\mathrm{b}^{*}$ components of color images.

The DoG operator model of center-surround receptive field would not be appropriate in this case. From the findings reported in [9] and [10], we infer that the operator to be applied on $\mathrm{a}^{*}$ and $b^{*}$ components of color images should represent a low-pass filter and not a bandpass filter like the DoG operator. Therefore, we suggest that instead of the DoG operator $g_{1}-g_{2}$, the operator $g_{1}+g_{2}$ be considered. Hence, we have

$$
O(x, y)=C(x, y)+S(x, y)=f *\left(g_{1}+g_{2}\right)
$$

where $O$ is now the output of the operator $g_{1}+g_{2}$, which is applied on the $\mathrm{a}^{*}$ and $\mathrm{b}^{*}$ color components of the underlying color image and $f$ is a opponent color component. Note that as mentioned in Section III-A.1, both the Gaussian functions $g_{1}$ and $g_{2}$ are low-pass filters and, hence, the operator $g_{1}+g_{2}$ is also a low-pass filter. We shall now see how the operator $g_{1}+$ $g_{2}$ mimics the center-surround retinal receptive field in coloropponent organization. Consider the operation $C+S$ (notation $(x, y)$ dropped for simplicity) in red-green $\left(\mathrm{a}^{*}\right)$ opponent color 


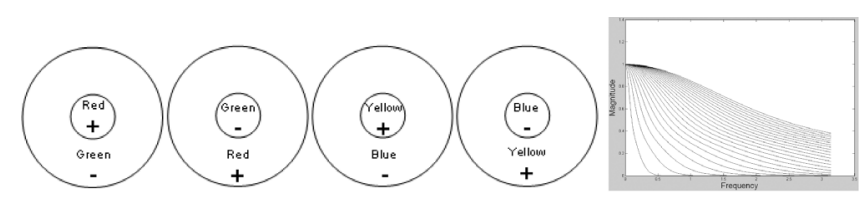

(a)

(b)

Fig. 5. Pictorial representation of some vision related phenomena of the retina during perception of chromatic signals $\mathrm{a}^{*}$ and $\mathrm{b}^{*}$. (a) Center-surround retinal receptive fields in color opponent organization. (b) Multiple low-pass channels.

component, where we have $C \equiv R_{C}-G_{C}$ and $S \equiv R_{S}-G_{S}$ and, hence, we get

$$
C+S \equiv\left(R_{C}-G_{S}\right)+\left(-G_{C}+R_{S}\right)
$$

where $R_{C}, G_{C}, R_{S}$, and $G_{S}$ represent red component center, green component center, red component surround and green component surround, respectively. From (28), it is evident that we get a red component center with an antagonistic green component surround and a green component center with an antagonistic red component surround. Similarly, considering the operation $C+S$ in yellow-blue ( $\left.\mathrm{b}^{*}\right)$ opponent color component, where we have $C \equiv Y_{C}-B_{C}$ and $S \equiv Y_{S}-B_{S}$ and, hence, we get

$$
C+S \equiv\left(Y_{C}-B_{S}\right)+\left(-B_{C}+Y_{S}\right)
$$

where $Y_{C}, B_{C}, Y_{S}$, and $B_{S}$ represent yellow component center, blue component center, yellow component surround and blue component surround, respectively. From (28), it is evident that we get a yellow component center with an antagonistic blue component surround, a blue component center with an antagonistic yellow component surround. The four center-surround retinal receptive field mimicked by applying $g_{1}+g_{2}(C+S)$ on the $\mathrm{a}^{*}$ and $\mathrm{b}^{*}$ opponent color components is given in Fig. 5(a).

We calculate the local band-limited contrast in the opponent color components of a color image as

$$
c(x, y)=\frac{O(x, y)-\bar{f}(x, y)}{S(x, y)}
$$

where $\bar{f}(x, y)$ represents local average calculated considering a $k \times k$ window, where $k \gg \sigma_{g_{2}}$. The quantity $O(x, y)-$ $\bar{f}(x, y)$ approximately represents output from a bandpass filter with passband almost same as that of the low-pass filter $g_{1}+$ $g_{2}$ except at zero frequency, where the passband magnitude is zero. The quantity $|c(x, y)|$ gives the contrast measure at a pixel $(x, y)$.

Similar to Section III-A.1, we consider here the multiple values of $\sigma_{g_{1}}$ given in (23) in order to mimic the presence of multiple frequency channels in the retina. We consider the same values of $\sigma_{g_{1}}$ as used in Section III-A.1, because it is reported in [10] that the retinal responses to chromatic and achromatic signals are of the same order. Multiple standard deviation values of $g_{1}$ give multiple spatial low-pass filters $g_{1}+g_{2}$ having different passbands and, hence, we get multiple $|c(x, y)|$ values which we represent as $\left|c_{\sigma_{g_{1}}}(x, y)\right|$. The passbands (frequency channels) of 1-D equivalents of the 24 low-pass filters (normalized such that the maximum passband magnitude value is unity) corresponding to the 24 values of $\sigma_{g_{1}}$ are shown in Fig. 5(b).

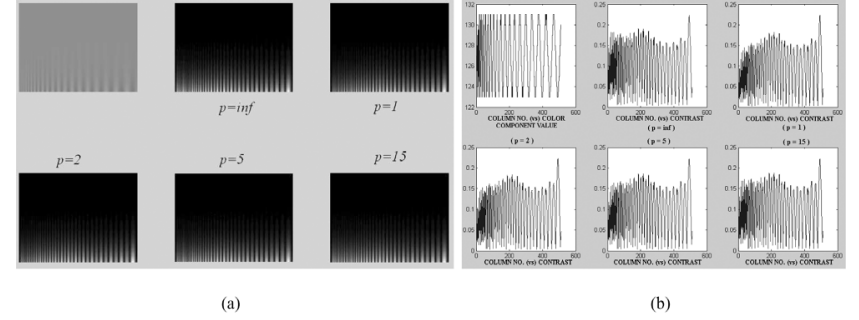

Fig. 6. Contrast measures obtained in an image representing an opponent color component, which has sinusoidal pattern with spatial frequency decreasing from left to right, considering different values of $p ; p=\infty, 1,2,5,15$ [top-middle to bottom-right]. (a) Image with sinusoidal pattern and its different contrast measures (normalized to the range $[0,1]$ ) at all pixels. (b) 1 -D color component value and contrast profiles across columns.

Similar to Section III-A.1, we need to combine the contrast measures $\left|c_{\sigma_{g_{1}}}(x, y)\right|$ in order to get a single contrast value at a pixel $(x, y)$ and we perform the combination considering findings from the study of sub- and supra-threshold contrast perception in the case of chromatic signals reported in [12].

In the case of chromatic signals, it has been found that during subthreshold contrast perception, contrast sensitivity is lower when the underlying pattern has high spatial frequencies compared to when the pattern has low spatial frequencies and contrast sensitivity characteristics show very little variation across spatial frequencies during supra-threshold contrast perception [12]. It is very interesting to find that similar to the case of achromatic signal, $\mathrm{L}^{\mathrm{P}}$-norm can be used in the case of chromatic signals in order to perform the combination of all the $\left|c_{\sigma_{g_{1}}}(x, y)\right| \mathrm{s}$ in such a way that the aforesaid phenomena about sub- and supra-threshold contrast perception are mimicked. We combine the measures $\left|c_{\sigma_{g_{1}}}(x, y)\right|, \forall \sigma_{g_{1}}$ as follows:

$$
c_{\zeta}(x, y)=\left(\sum_{\sigma_{g_{1}}}\left(0.5 \times\left|c_{\sigma_{g_{1}}}(x, y)\right|\right)^{p}\right)^{\left(\frac{1}{p}\right)}
$$

where $p \geq 1$ and $\zeta$ represents one of the two opponent color components $\mathrm{a}^{*}(R-G)$ and $\mathrm{b}^{*}(Y-B)$. In the previous equation, the scaling by 0.5 ensures that the maximum magnitude value of the passband of the underlying low-pass filter is unity [see Fig. 5(b)]. The measure $c_{\zeta}(x, y)$ gives the contrast measure in the opponent color component $f$ of the underlying color image at a pixel $(x, y)$. When $p=1\left(\mathrm{~L}^{1}\right.$ norm $)$ is considered in (24), the subthreshold contrast perception is best mimicked and when $p=\infty$ (maximum norm) is considered in (24), the supra-threshold contrast perception is best mimicked. Fig. 6(a) shows an image, which represents an opponent color component of a color image, having sinusoidal pattern with varying magnitude (across rows) and spatial frequency (across columns), and the contrast measure $c_{\zeta}$ obtained at all pixels using different values of $p$, and Fig. 6(b) shows their corresponding 1-D profiles across columns.

Notice the same wavy envelope in all the one dimensional profiles of the contrast measure $c_{\zeta}$ obtained using different values of $p$. The wavy envelope is due to the usage of the quantity $\bar{f}(x, y)$ in (29) in order to make the underlying passband's magnitude zero at zero frequency and, hence, we can ignore the 
wavy envelope while analyzing Fig. 6(b). Observe the cases of $p=1$ and $p=2$ in Fig. 6(b), where contrast sensitivity is lower when the spatial frequency is high, and the cases of $p=15$ and $p=\infty$, where contrast sensitivity hardly varies across spatial frequencies. Hence, it is evident in Fig. 6 that with increase in the value of $p$, the contrast sensitivity characteristics gradually changes from that of subthreshold color contrast perception to supra-threshold color contrast perception. Color contrast levels in a color image may vary from the minimum contrast level required for detection of a pattern to much higher levels and, hence, the choice of $p$ is not obvious like in Section III-A.1.

Once the contrasts in $\mathrm{L}^{*}$ (achromatic signal), $\mathrm{a}^{*}$ and $\mathrm{b}^{*}$ (chromatic signals) components corresponding to an image are calculated, the contrast measure $c_{I}$ of the image at a pixel $(x, y)$ is obtained as follows:

$$
c_{I}(x, y)=c_{L}(x, y)+c_{(R-G)}(x, y)+c_{(Y-B)}(x, y)
$$

where $c_{L}$ is the contrast in $\mathrm{L}^{*}$ (lightness) component, $c_{(R-G)}$ and $c_{(Y-B)}$ are the contrasts in the opponent color components $\mathrm{a}^{*}$ and $\mathrm{b}^{*}$. Note that we consider the same value of $p$ while using $\mathrm{L}^{\mathrm{p}}$ norm in the calculation of contrasts due to the achromatic and chromatic signals corresponding to an image. It is worthwhile to mention here that a few crude attempts of contrast measurement in grayscale and color images based only upon the DoG modeling of the retinal receptive field have been made in [16] and [17].

\section{B. Quantitative Evaluation of Contrast Enhancement}

As mentioned in Section I, contrast enhancement makes various contents of images easily distinguishable through suitable increase in contrast. In this paper, we shall measure increase of contrast at an image pixel due to the application of a contrast enhancement technique as follows:

$$
\chi(x, y)=\frac{\overline{c_{I}}+c_{I_{E}}(x, y)}{\overline{c_{I}}+c_{I}(x, y)}
$$

where $I$ represents the underlying image, $I_{E}$ represents the contrast enhanced image and $\overline{c_{I}}$ represents the average contrast in $I$. The term $\overline{c_{I}}$ is considered in (32) to ensure that pathological cases such as $\chi(x, y)=0$ and $\chi(x, y)=\infty$ do not occur, assuming that $\overline{c_{I}}$ is always nonzero.

Although increase in image contrast makes its contents more distinguishable, the pleasingness of the image might suffer in the process [18], [19]. Hence, suitability of contrast enhancement through increase in contrast lies in not decreasing the pleasingness of the image. In fact, nonreference image quality measures such as the most eye-pleasing sharpness [19] have been used to check the suitability of image enhancement. From [19] we infer that if the contrast in both the homogeneous and the heterogeneous areas of the image under consideration is increased by similar amounts (in terms of $\chi$ ) then the pleasingness of the image does not decrease. On the other hand, if the contrast in either the homogeneous or the heterogeneous areas is increased much more than the other then the pleasingness of the image will deteriorate. Although it is not mentioned in [19], it is obvious that the contrast in the homogeneous areas could be increased only by amounts (in terms of $\chi$ ) such that clutters do not appear.
We calculate the increase of contrast in heterogeneous and homogeneous areas respectively as

$$
\begin{aligned}
& \chi^{\text {he }}(x, y)=\alpha(x, y) \times \chi(x, y) \\
& \chi^{\text {ho }}(x, y)=\left(\alpha^{1}-\alpha(x, y)\right) \times \chi(x, y)
\end{aligned}
$$

where

$$
\alpha(x, y)=\exp \left(-\frac{\left(\tilde{c_{I}}(x, y)-1\right)^{2}}{2 \Sigma^{2}}\right)
$$

and $\alpha^{1}=1+\exp \left(-\left((-1)^{2}\right) /\left(2 \Sigma^{2}\right)\right)$. In (35), $\tilde{c_{I}}(x, y)=$ $\left(c_{I}(x, y)\right) /\left(\max _{\forall(x, y)} c_{I}(x, y)\right)$ and, hence, $\tilde{c_{I}}(x, y) \in[0,1]$. The quantity $\alpha(x, y)$ gives the belongingness (membership) of the pixel $(x, y)$ to heterogeneous areas and $\alpha^{1}-\alpha(x, y)$ gives the belongingness to homogeneous areas. Note that, we take $\Sigma=0.455$, as in such a case, a pixel, where $\tilde{c_{I}}$ is exactly at the middle $(0.5)$ of the two extremes 0 and 1 , belongs almost equally to heterogeneous and homogeneous areas.

In this paper, we shall perform quantitative evaluation of contrast enhancement considering that higher value of $\chi$ signifies better contrast enhancement provided that $\chi^{\text {he }} \approx \chi^{\text {ho }}$ and it is visually confirmed that clutter has not appeared.

\section{EXPERIMENTAL RESULTS}

In this section, we provide experimental results in order to demonstrate the effectiveness of the proposed automatic exact histogram specification technique in comparison to a few existing methods both qualitatively and quantitatively. Qualitative analysis is performed by visual comparison and as presented in Section III-B, quantitative analysis is carried out using the proposed method of measuring image contrast. We consider the use of the proposed exact histogram specification technique for global and local contrast enhancement of grayscale images. These enhancement techniques could be easily extended to color images by using the approach in [20].

We shall now consider global and local contrast enhancement of images separately and compare the use of the proposed exact histogram specification technique with the different existing methods considered under each category.

\section{A. Global Contrast Enhancement}

Global contrast enhancement is aimed at increasing the overall contrast of an image. Here, we apply the proposed exact histogram specification (proposed EHS) technique on global histograms of images in order to perform global contrast enhancement. The performance of the proposed technique is compared to that of exact histogram equalization (EHE) [1] and gray-level grouping (GLG) [15], which are also applied on global histograms.

Consider the grayscale image given in Fig. 7(a). The global histogram considered here is the gray-level histogram of the image. The images in Fig. 7(b)-(d) are obtained by performing global contrast enhancement using the proposed EHS, EHE and GLG techniques, respectively. As can be seen the overall contrast is higher in the image in Fig. 7(b), whereas, the brightness seems to be higher in the image in Fig. 7(d). Fig. 7(e)-(g), respectively show the average $\chi, \chi^{\text {he }}$ and $\chi^{\text {ho }}$ (average taken over all the pixels in the image) obtained when the three techniques 


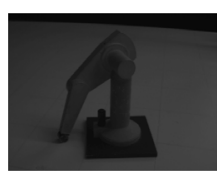

(a)

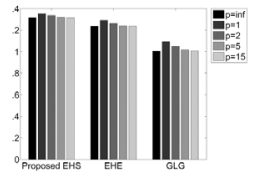

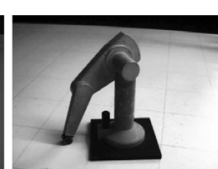

(b)

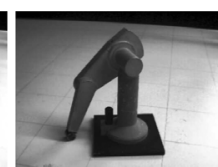

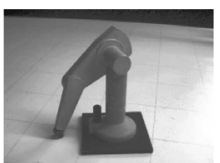

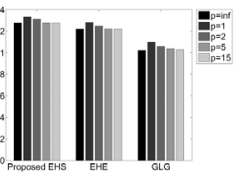

(g)
Fig. 7. Global contrast enhancement of grayscale images using the proposed EHS, EHE and GLG techniques. (a) Image. (b) Proposed EHS. (c) EHE. (d) GLG. (e) $\chi$ values. (f) $\chi^{\text {he }}$ values. (g) $\chi^{\text {ho }}$ values.

are applied on the image in Fig. 7(a) and the different values of $p$ are considered. It is evident that the average $\chi$ is higher when the proposed EHS is used and we have average $\chi^{\text {he }} \approx$ average $\chi^{\text {ho }}$ in all the three cases.

\section{B. Local Contrast Enhancement}

Local contrast enhancement is aimed at increasing contrast in local neighborhoods in images in order to reveal minute details. We consider the contrast limited adaptive (local) enhancement approach pioneered in [21], in order to carry out local contrast enhancement using the EHE and the proposed EHS techniques, which are applied to histograms calculated within local neighborhoods in images. The contrast limited approach is considered as it helps in avoiding over enhancement, which is usual in local histogram based enhancement techniques. The control of enhancement is achieved by limiting the slope of the mapping function corresponding to histogram equalization/specification operation [21]. Note that, unlike histogram equalization/specification, an underlying mapping function is not used in the case of EHE and the proposed EHS [1]. However, once the EHE or the proposed EHS has been performed the underlying mapping function can be calculated. We first do so, where we linearly spread the underlying points (values of the argument of the function) wherever required to ensure that the slope calculated is not $\infty$ at any point. We then limit the slope of the mapping function (as described in [21]) with the maximum allowed slope being $s l_{a}+\left(s l_{m}-s l_{a}\right)\left(s l_{a}\right) /\left(s l_{m}\right)$, where $s l_{a}$ and $s l_{m}$ are the average and maximum slope over all the points. The modified mapping function is then used to redo the EHE and the proposed EHS to get the contrast limited adaptive EHE (CLAEHE) and the proposed contrast limited adaptive EHS (proposed CLAEHS), respectively. The performance of the proposed CLAEHS is compared to that of CLAEHE, local standard deviation distribution modeling based unsharp masking (MUM) proposed in [22] and human visual properties based algorithm RACE given in [23], as all of them are adaptive (local) contrast enhancement techniques.

Consider the grayscale image given in Fig. 8(a). The images in Fig. 8(b)-(e) are obtained by performing local contrast enhancement using the proposed CLAEHS, CLAEHE, MUM, and RACE techniques, respectively. As can be seen, details such as that in the top-right of the image has been revealed better in

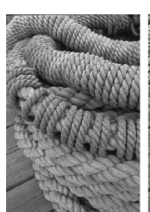

(a)

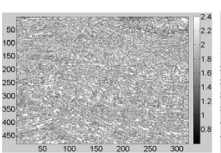

(g)

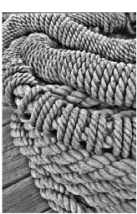

(b)

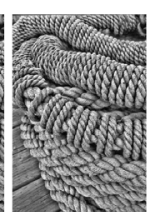

(c)

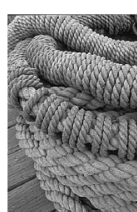

(d)

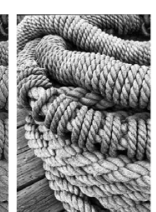

(e)

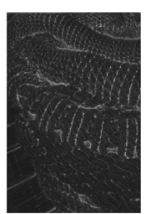

(f)

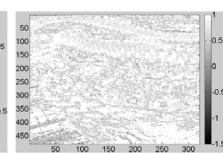

(j)
Fig. 8. Local contrast enhancement of a grayscale image using the proposed CLAEHS, CLAEHE, MUM, and RACE techniques. (a) Image. (b) Prop. CLAEHS. (c) CLAEHE. (d) MUM. (e) RACE. (f) $\alpha$. (g) $\chi_{\text {ClAeHs }}$. (h) $\left(\chi_{\text {ClAehs }}-\chi_{\text {ClAehe }}\right)$. (i) $\left(\chi_{\text {ClAehs }}-\chi_{\text {MUM }}\right)$. (j) $\left(\chi_{\text {Claehs }}-\right.$ $\left.\chi_{\mathrm{RACE}}\right)$.

the image in Fig. 8(b). As the aim of local contrast enhancement is to increase contrast in local neighborhoods, we do not consider average $\chi, \chi^{\text {he }}$, and $\chi^{\text {ho }}$ values for analysis as they only represent overall contrast increase. Instead, we consider the contour maps in Fig. $8(\mathrm{~g})-(\mathrm{j})$ showing $\chi_{\mathrm{CLAEHS}},\left(\chi_{\mathrm{CLAEHS}}-\right.$ $\left.\chi_{\mathrm{CLAEHE}}\right),\left(\chi_{\mathrm{CLAEHS}}-\chi_{\mathrm{MUM}}\right)$ and $\left(\chi_{\mathrm{CLAEHS}}-\chi_{\mathrm{RACE}}\right)$, respectively, where the subscript represents the associated technique and $p=1$. We see that $\chi$ is higher at most of the pixels when the proposed CLAEHS is used. The image in Fig. 8(f) shows the membership $\alpha([0,1] \rightarrow$ [black, white] $)$ that gives the belongingness of pixels to heterogeneous areas. Considering this image, we find that in local neighborhoods, $\chi$ is slightly higher at pixels in either heterogeneous or homogeneous area compared to pixels in the other when the proposed CLAEHS, CLAEHE, and RACE are used, which is not significantly evident when MUM is used. Hence, the image in Fig. 8(d) appears more pleasing.

\section{CONCLUSION}

An automatic exact histogram specification technique has been proposed in this paper and it has been used for global and local contrast enhancement of images. The desired histogram has been obtained by first subjecting the image histogram to a modification process that increases the overall discriminablity among samples in the histogram and then by maximizing a measure that represents increase in information and decrease in ambiguity, which are contradictory indicators of contrast enhancement. Based upon qualitative and quantitative analyses, the proposed automatic exact histogram specification technique has been found effective in enhancing contrasts of images in comparison to a few existing methods.

In order to perform the quantitative analysis, a new method of measuring image contrast based on local band-limited approach and antagonistic center-surround retinal receptive field model has been devised in this paper. Various evidences from physiological studies corresponding to contrast perception along with the existence of multiple frequency channels in the visual system have been considered. In accordance to them, the proposed method has been allowed to work at multiple scales (frequency bands) and combination of the contrast measures obtained at the different scales has been performed 
using $\mathrm{L}^{\mathrm{p}}$-norm. Suitable functions involving image contrast measured using the proposed approach have been employed for the evaluation of contrast enhancement. Although the proposed approach of contrast measurement has been used for evaluation of contrast enhancement, the approach is general, and hence, it can be used for other image processing tasks.

\section{REFERENCES}

[1] D. Coltuc, P. Bolon, and J. M. Chassery, "Exact histogram specification,” IEEE Trans. Image Process., vol. 15, no. 5, pp. 1143-1152, May 2006.

[2] D. T. Cobra, "A generalization of the method of quadratic hyperbolization of image histograms," in Proc. 38th Midwest Symp. Circuits Syst., Aug. 1995, vol. 1, pp. 141-144.

[3] C. E. Shannon, "A mathematical theory of communication," Bell Syst. Tech. J., vol. 27, pp. 379-423, 1948.

[4] D. Sen and S. K. Pal, "Generalized rough sets, entropy, and image ambiguity measures," IEEE Trans. Syst., Man, Cybern. B, Cybern., vol. 39, no. 1, pp. 117-128, Feb. 2009.

[5] E. Peli, "Contrast in complex images," J. Opt. Soc. Amer. A, vol. 7, no. 10, pp. 2032-2040, 1990.

[6] E. Kandel, J. Schwartz, and T. Jessell, Principles of Neural Science, 4th ed. New York: McGraw-Hill, 1994, ch. 25-27, pt. V.

[7] R. W. Rodieck and J. Stone, "Analysis of receptive fields of cat retinal ganglion cells," J. Neurophysiol., vol. 28, no. 5, pp. 833-849, 1965.

[8] A. K. Jain, Fundamentals of Digital Image Processing. New Delhi, India: Prentice-Hall, 2001.

[9] K. T. Mullen, "The contrast sensitivity of human colour vision to redgreen and blue-yellow chromatic gratings," J. Physiol., vol. 359, pp. 381-400, 1985.

[10] J. M. Rovamo, M. I. Kankaanpää, and H. Kukkonen, "Modelling spatial contrast sensitivity functions for chromatic and luminance-modulated gratings," Vis. Res., vol. 39, no. 14, pp. 2387-2398, 1999.

[11] M. A. Georgeson and G. D. Sullivan, "Contrast constancy: Deblurring in human vision by spatial frequency channels," J. Physiol., vol. 252, pp. 627-656, 1975.

[12] R. L. P. Vimal, "Spatial color contrast matching: Broad-bandpass functions and the flattening effect," Vis. Res., vol. 40, no. 23, pp. 3231-3243, 2000.

[13] R. L. DeValois and K. K. DeValois, Spatial Vision. London, U.K.: Oxford Univ. Press, 1990.

[14] D. Sen and S. K. Pal, "Feature space based image segmentation via density modification," in Proc. IEEE Int. Conf. Image Process., 2009, pp. 4017-4020.

[15] Z. Y. Chen, B. R. Abidi, D. L. Page, and M. A. Abidi, "Gray-level grouping (GLG): An automatic method for optimized image contrast enhancement-part I: The basic method," IEEE Trans. Image Process., vol. 15, no. 8, pp. 2290-2302, 2006.

[16] Y. Tadmor and D. J. Tolhurst, "Calculating the contrasts that retinal ganglion cells and LGN neurones encounter in natural scenes," Vis. Res., vol. 40, no. 22, pp. 3145-3157, 2000.

[17] A. Rizzi, G. Simone, and R. Cordone, "A modified algorithm for perceived contrast measure in digital images," in Proc. 4th Eur. Conf. Color Graph., Imag. Vis., 2008, vol. 4, pp. 249-252.

[18] T. J. W. M. Janssen, “Computational image quality," Ph.D. dissertation, Tech. Univ. Eindhoven, Eindhoven, The Netherlands, 1999.

[19] W. S. Lin, Y. L. Gai, and A. A. Kassim, "Perceptual impact of edge sharpness in images," in IEE Proc. Vis. Image Signal Process., 2006, vol. 153 , no. 2 , pp. $215-223$.

[20] S. K. Naik and C. A. Murthy, "Hue preserving color image enhancement without gamut problem," IEEE Trans. Image Process., vol. 12, no. 12 , pp. 1591-1598, Dec. 2003.
[21] S. M. Pizer, E. P. Amburn, J. D. Austin, R. Cromartie, A. Geselowitz, T. Greer, B. T. H. Romeny, and J. B. Zimmerman, "Adaptive histogram equalization and its variations," Comput. Vis. Graph. Image Process., vol. 39, no. 3, pp. 355-368, 1987.

[22] D. C. Chang and W. R. Wu, "Image contrast enhancement based on a histogram transformation of local standard deviation," IEEE Trans. Med. Imag., vol. 17, no. 4, pp. 518-531, 1998.

[23] E. Provenzi, C. Gatta, M. Fierro, and A. Rizzi, "A spatially variant white-patch and gray-world method for color image enhancement driven by local contrast," IEEE Trans. Pattern Anal. Mach. Intell., vol. 30 , no. 10 , pp. $1757-1770$, Oct. 2008.

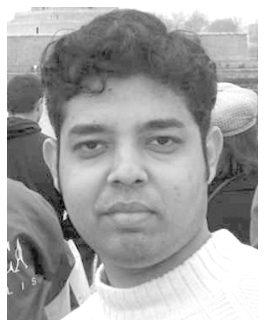

Debashis Sen (S'05-M'06) received the M.A.Sc. degree in electrical engineering from Concordia University, Montreal, QC, Canada, in 2005, and the B.Engg. (Hons.) degree in electronics and communication engineering from the University of Madras, India, in 2002, and is currently working towards the Ph.D. degree at the Center for Soft Computing Research, Indian Statistical Institute, Kolkata, India.

He worked at the Center for Signal Processing and Communications (CENSIPCOM) and with the Video Processing and Communications Group at Concordia University from 2003 to 2005 . His research interests are in image and video processing, probability theory, and soft computing.

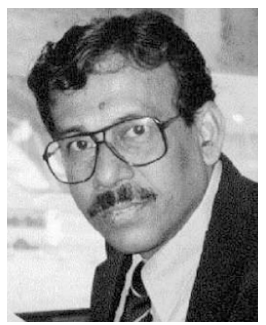

Sankar K. Pal (M'81-SM'84-F'93) received the $\mathrm{Ph}$.D. degree in radio physics and electronics from the University of Calcutta, India, in 1979, and the $\mathrm{Ph} . \mathrm{D}$. degree along with a Diploma of Imperial College (DIC) from Imperial College, University of London, U.K., in 1982.

He is a Distinguished Scientist and former Director of the Indian Statistical Institute. Currently, he is also a J. C. Bose Fellow of the Government of India. He founded the Machine Intelligence Unit and the Center for Soft Computing Research: A National Facility in the Institute in Calcutta. He worked at the University of California, Berkeley and the University of Maryland, College Park from 1986 to 1987; the NASA Johnson Space Center, Houston, Texas from 1990 to 1992 and 1994; and in US Naval Research Laboratory, Washington DC in 2004. Since 1997, he has been serving as a Distinguished Visitor of IEEE Computer Society for the Asia-Pacific Region, and held several visiting positions in Italy, Poland, Hong Kong, and Australian universities. He is a coauthor of fifteen books and more than 300 research publications in the areas of pattern recognition, machine learning, image processing, data mining, web intelligence, soft Computing and bioinformatics.

Prof. Pal is a Fellow of the Academy of Sciences for the Developing World (TWAS), Italy, International Association for Pattern Recognition, International Association of Fuzzy Systems, and all the four National Academies for Science/Engineering in India. He has received the 1990 S. S. Bhatnagar Prize (which is the most coveted award for a scientist in India), and many prestigious awards in India and abroad including the 1999 G. D. Birla Award, 1998 Om Bhasin Award, 1993 Jawaharlal Nehru Fellowship, 2000 Khwarizmi International Award from the Islamic Republic of Iran, 2000-2001 FICCI Award, 1993 Vikram Sarabhai Research Award, 1993 NASA Tech Brief Award (USA), 1994 IEEE Trans. NeURAL Networks Outstanding Paper Award (USA), 1995 NASA Patent Application Award (USA), 1997 IETE-R. L. Wadhwa Gold Medal, the 2001 INSA-S. H. Zaheer Medal, 2005-06 Indian Science Congress-P. C. Mahalanobis Birth Centenary Award (Gold Medal) for Lifetime Achievement, 2007 J. C. Bose Fellowship of the Government of India and 2008 Vigyan Ratna Award from Science and Culture Organization, West Bengal. 